\title{
Monitoring sea surface with SAR: studies and applications
}

\author{
W. Biamino ${ }^{1}$, F. Tataranni ${ }^{2}$, M. Borasi ${ }^{1}$, C. De Vecchi ${ }^{1}$ \\ \& P. Trivero ${ }^{1}$ \\ ${ }^{I}$ Life and Environment Science Department, \\ Eastern Piedmont University, Alessandria, Italy \\ ${ }^{2}$ INNOVA Consortium, Matera, Italy
}

\begin{abstract}
In recent decades, the Synthetic Aperture Radar (SAR) has been increasingly used as a powerful tool for sea surface monitoring thanks to its peculiar features (all weather, day and night monitoring). Among possible applications, SAR is widely used for oil spill detection and tracking on the sea surface because of its capabilities to evidence the wave damping induced by surface active slicks; it is also possible to measure natural phenomena such as wind stress or surface currents.

In recent years we have developed and tested algorithms for automatic oil spill detection and wind extraction from ERS and ENVISAT images. In this paper we first resume our experience, showing the present status and planned advances.

We also show some examples of operational applications, done in the framework of national and international projects. A special attention is paid to InterRisk project, where an open system architecture, based on noted GIS and web standards, has been set up in order to integrate and harmonise different kind of data (satellite, in-situ, model simulations) from different service providers in order to allow users an easy and effective access to the information needed.
\end{abstract}

Keywords: oil slicks, Synthetic Aperture Radar, GIS, web service.

\section{Introduction}

For several years remote sensing has been widely used for land and sea surface observation. Optical sensors are well tested tools to monitor land use, vegetation 
health or algal proliferation on the sea surface, although they have a major drawback: their use is possible only in the presence of sunlight and clear sky conditions, because optical and infrared radiation is adsorbed by clouds.

Active sensors such as SAR (Synthetic Aperture Radar) can overcome this problem: microwaves, generated by the satellite itself, are reflected back and measured, regardless of weather condition and sunlight (water vapour is transparent to microwaves). Spaceborne sensors can therefore measure on a 24/7 basis, being powerful tools to monitor both natural and man induced phenomena.

When analysing SAR images, the intensity of the backscattered signal (also known as Normalized Radar Cross-Section - NRCS) is strictly determined by the geometrical features of the water surface. Surfactant slicks, reducing water surface roughness, appear as dark areas and therefore can be easily detected. On this basis, SAR images have been used in the past for oil spill detection with different approaches: visual inspection by photo-interpreters or automatic techniques based on different principles: neural networks, statistical techniques, empirical measurements, etc.

Existing satellite SAR systems have already demonstrated their capability to detect oil spills in many sea areas, as reported by e.g. Espedal and Johannessen [1] and Alpers and Espedal [2]. In addition to oil spills, there are many others surface features (such as wind, current features, natural films, surface temperature patterns, rain cells) that provide radar signatures similar to oil, as shown by, for example, Beal et al. [3] and Espedal [4].

However, operational use of SAR is limited by some factors.

First, every satellite has a revisit time (the time interval between two consecutive passes on the same area), determined by orbit characteristics and latitude. For example, this interval could be as long as several days for ERS satellites on the Mediterranean Sea.

Another limiting factor is the spatial resolution. With large pixels, small slicks cannot be detected; moreover, slick detection close to the coastline can be difficult.

At last, real time access to various SAR data can be limited by practical restraints such as different data formats and distribution channels.

In recent years, European Space Agency (ESA) has set up the eoPortal [5], a web portal aimed to become a single access point to a large variety of information and services for Earth Observation, including satellite imagery. In the framework of eoPortal, the Service Support Environment (SSE) [6] implements an open service-oriented and distributed environment among users (service users and service providers), enabling the integration of satellite data, meteorological, oceanographic and GIS data. The SSE environment facilitates both service provision and orchestration, allowing each organisation to exploit the service know-how and data availabilities of the others, also for the creation of new services from a horizontal set of basic services supplied by multiple service providers.

In the framework of GMES (Global Monitoring for Environment and Security), a European initiative for the implementation of information services dealing with environment and security, European Commission finances a 
number of research projects aimed to set up pilot services in fields such as oceanography, precise mapping of land use, rapid mapping at times of emergency for the civil protection field or air quality monitoring.

InterRisk, a FP6-IST project addressing interoperables GMES services for environmental risk management in marine and coastal areas of Europe, is an example of such a project; several "basic" and "advanced" services are available by means of a web portal, integrated on ESA SSE.

\section{Oil spill detection with SAR}

Surfactants are responsible for sea wave damping and reflectivity modulation over a broad range of frequencies from the visible to the microwave regions of the spectrum. Measurements of slick-induced damping of short-gravity ocean waves excited by the wind provide useful data for the investigation and characterization of ocean microlayers on a thermodynamic basis. By means of a theoretical model one can infer the soluble or insoluble nature of the substance forming the film itself, the rheological parameters and the surface film fragmentation.

Among all monitoring ways, multi-frequency radar and SAR are powerful tools for the detection and characterization of substances forming sea surface film.

The basic mechanism involved is the normalised radar cross-section which, for incidence angles higher than $20^{\circ}$, is proportional to the spectral energy density of the sea waves having wavelength $\Lambda$ that obey the Bragg resonance condition:

$$
\Lambda=\frac{\lambda}{2 \sin \vartheta}
$$

where $\lambda$ is the radar wavelength and $\theta$ the incidence angle of radar beam. For low incidence angles the backscatter is due to specular reflection. The sea waves, which are Bragg resonant with microwaves employed by the SAR systems, fall in the short gravity wave region where is found a maximum in the ratio between spectra measured in pure water and in water covered by film.

The ability of multi-frequency SAR to characterise surface films has been tested with data from many experimental campaigns, showing that multifrequency SAR is an ideal instrument to monitor sea surface substances, since SAR data contain information about the spectral components affected by damping $[7,8]$.

The previously cited experiences show the feasibility of an automatic detection of surface slicks on SAR images. Since today, various approaches have been used for detection and characterisation with the aim to identify oil slicks. In particular, a probabilistic approach has been developed and tested, using statistical information (physical and geometrical characteristics) obtained from a test dataset of recognised measurements of both oil spill and natural features. Previous studies evidences a number of typical slick characteristics ("slick signature"); for each selected dark area the following quantities are evaluated: 
Perimeter (P); Area (A); Average NRCS inside the dark area (SIGMAI); Average NRCS in a limited area outside the dark area (SIGMAO); NRCS Dark Area Standard Deviation (DASD); NRCS Outside dark area Standard Deviation (OSD); Gradient (GRD) of the NRCS across the dark area perimeter; Form Factor (FRM): the dispersion of dark area pixels from its longitudinal axis. And derived from these: Perimeter to Area ratio (P/A); Intensity Ratio (IRT) between average NRCS inside and outside the dark area; NRCS Standard Deviations Ratio (SDR) inside and outside the dark area; Ratio between NRCS Intensity and its Standard Deviation Inside the dark area (RISDI); Ratio between NRCS Intensity and its Standard Deviation Outside the dark area (RISDO); RISDI to RISDO Ratio (IOR).

Parameters are evaluated for dark areas on SAR images, then combined with statistical techniques in order to obtain a classification $[9,10]$.

\section{Marine features extraction}

As previously stated, gravity-capillary (Bragg) waves are the dominant contribution to the radar backscatter. It is also known that there exists a relationship between the sea surface wind and such waves. Therefore, the centimetre-wavelength radars can provide in principle sea-surface wind vector information, and as such, a wind-to-backscatter relationship exists. The latter is generally referred to as the geophysical model function (GMF).

Several attempts, theoretical and empirical, have been made to model a GMF. It is possible to demonstrate that the backscattering coefficient according to wind speed, wind direction and the angle of incidence. A well tested approach is CMOD, developed for the scatterometer on board of ERS satellites but also useful for wind extraction from SAR data; the present version is CMOD5 [11]

Scatterometers can directly evaluate wind vectors by combining multiple measures, from different points of view, on the same sea region; in order to evaluate wind speed from SAR images, external information about wind direction is needed. This can be done by using wind direction from mesoscale atmospheric model or by applying mathematical techniques such as Fourier analysis, in order to evidence periodic features induced by wind. Wavelet analysis seems to be a good choice for wind direction estimation, even in absence of periodic signatures [12].

\section{Future advances}

During several years, $\mathrm{C}$ band SAR data have been the most popular choice for monitoring and detecting oil spills, especially because C-band is less adsorbed by rain than other bands. On the other side, $\mathrm{X}$-band seems to be more effective than L- or C-band [13].

In last years two new sensors have become available: the German Terrasar-X and the Italian Cosmo - SkyMed (COnstellation of Small Satellites for Mediterranean basin Observation), both in X - band. These new sensors supply images at higher spatial resolution (up to $1 \mathrm{~m} /$ pixel) and revisit time. 
COSMO-SkyMed is a 4-spacecraft constellation for Earth observation, designed by Italian Space Agency; first and second have been launched yet. Each satellite is equipped with SAR instruments [14]. The nominal repeat cycle is 16 days but the full constellation should have a revisit time of a few hours on a global scale [15]. The X-band instrument has a swath between 10 and $200 \mathrm{~km}$ (depending on support mode) and a total Field of Regard of $1300 \mathrm{~km}$ in the cross-track direction. The operational modes are summarized in [16].

The wide availability of instruments and data makes the SAR as a useful tool for water quality monitoring system. The analysis of radar backscattering at different frequencies, together with the time evolution from images acquired on the same area in different but close times, can integrate the information presently provided by "classic" well tested slick detection procedures [17].

\section{$5 \quad$ Web tools for data distribution}

Oil slick detection data have been utilised into pilot systems, in order to test the possibility to integrate different kind of data (satellite images, oil slick detection, model outputs) in a single framework with the aim to allow users to obtain all information from a single source, regardless of data providers and formats.

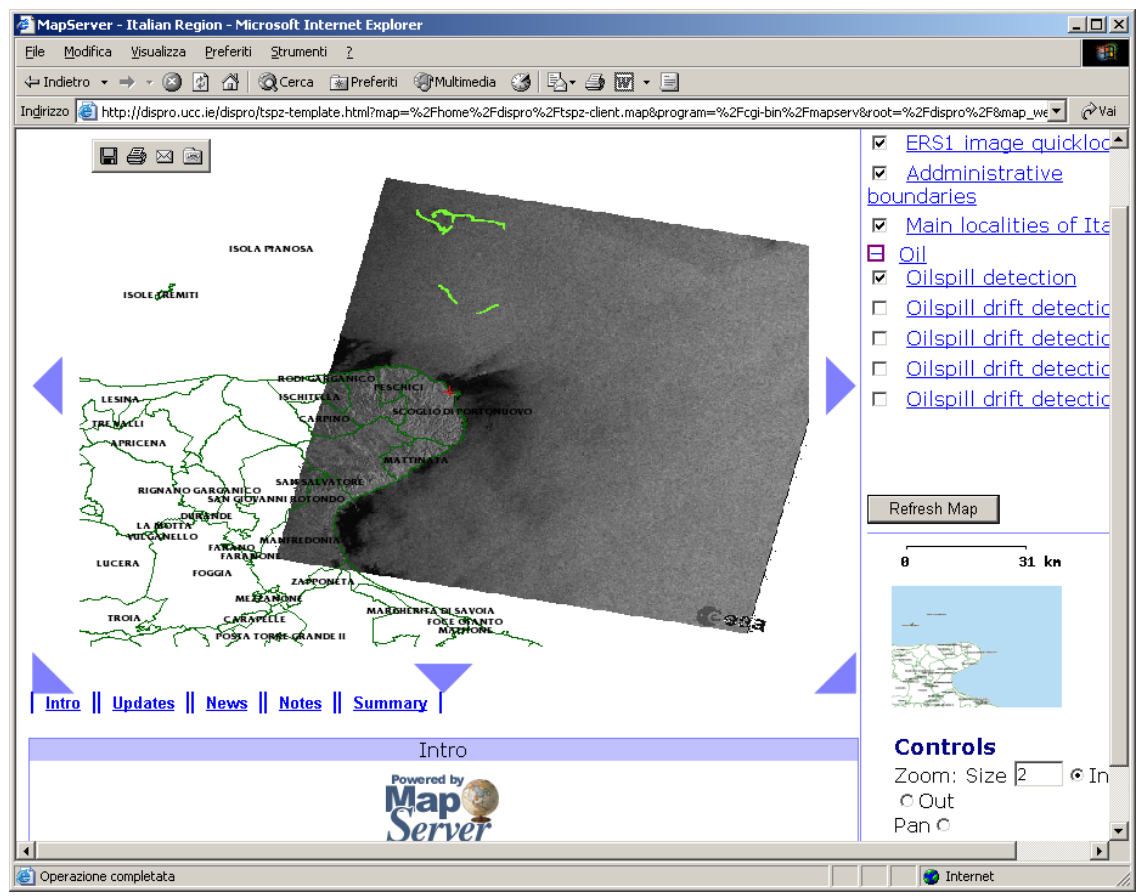

Figure 1: Sample DISPRO screenshot. 
The first attempt was made with DISMAR (Data Integration System for Marine Pollution and Water Quality) project, who lasted from 2002 to 2005. A web based distributed system, called DISPRO, was implemented in order to integrate and distribute multi source data and metadata, stored on a set of distributed computer nodes. DISPRO was based on open source tools, such as Apache web server and UMN Mapserver for GIS data distributed with WMS (Web Map Server) protocol [18].

DISPRO capabilities have been demonstrated on various European regions, showing its capabilities to deal with different kind of data (meteorological information, oil slicks, algae blooms, etc.). In figure 1, a sample DISPRO screenshot shows the outline of an oil slick, superimposed to SAR quicklook and administrative boundaries.

DISMAR concept has been developed and expanded on InterRisk (Interoperable GMES Services for Environmental Risk Management in Marine and Coastal Areas of Europe) project, started in 2006.

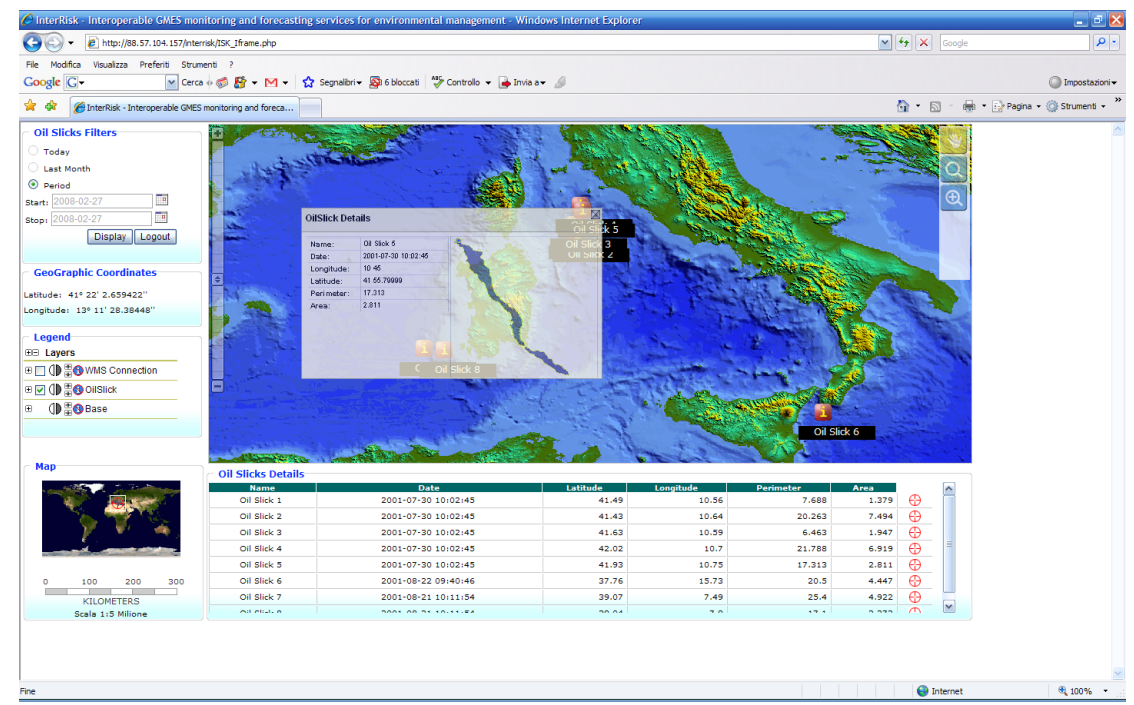

Figure 2: $\quad$ Screenshot of InterRisk regional portal for Italy.

While DISPRO was designed as a standalone tool, InterRisk pilot system is compatible with the ESA SSE environment, facilitating both service provision and orchestration, allowing each organisation to exploit the service know-how and data availabilities of the others, also for the creation of new services from a horizontal set of basic services supplied by multiple service providers.

Moreover, InterRisk pilot system is able to deal with different web protocols, overcoming the limitations of many present systems: instead of a basic GIS manipulation of raster maps (zoom, pan, select region of interest, return to original extent), InterRisk pilot system allows users to directly access and use data for more powerful applications, such as data merging and time series visualisation. 
The InterRisk pilot system and services will be validated by users responsibles for crisis management in case of oil spills, harmful algal blooms and other marine pollution events, in Norwegian, UK/Irish, French, German, Polish and Italian coastal waters.

Figure 2 shows a screenshot from the Italian "regional portal", e.g. the page from data provider before the integration into SSE. Figure 3 shows how vector data (the oil slick contour) is visualised.

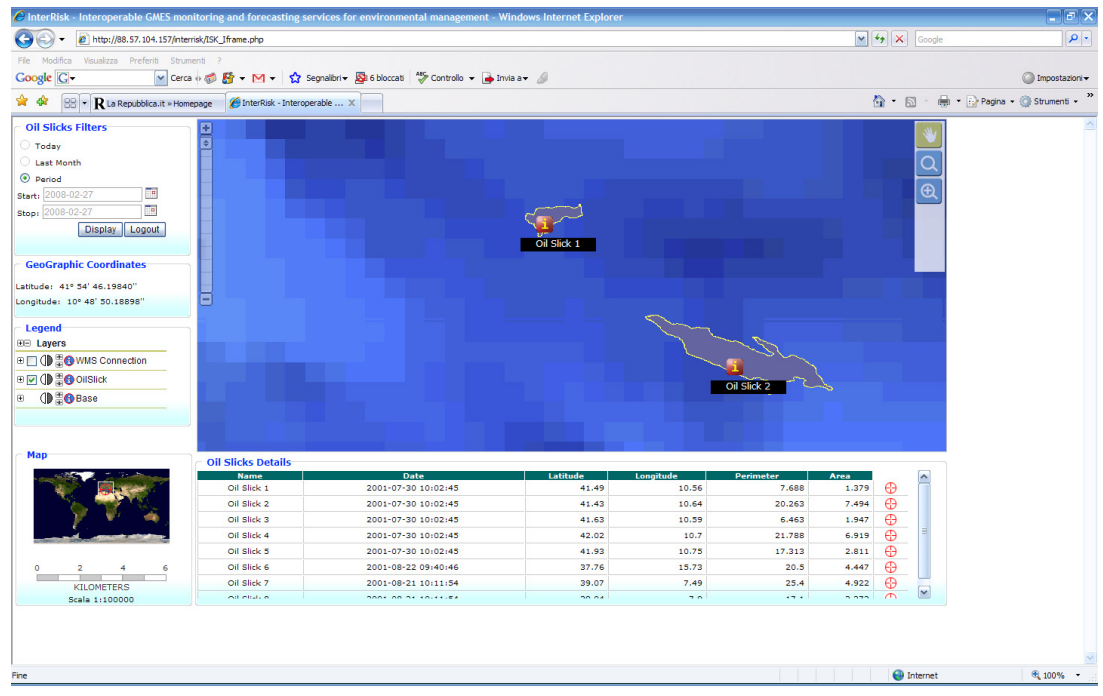

Figure 3: $\quad$ Vector data visualisation.

The Italian node, managed by INNOVA, is based on UMN Mapserver, configured to distribute Data And Metadata Repositories content (filled with test data) via WFS and WMS protocols. WFS interface has been used to register Italian-Water regional service on SSE portal. The WWW Regional Portal has been implemented Using PHPMapscript and KaMap extensions.

\section{Conclusions}

The usefulness of SAR data for sea surface monitoring, well assessed since several years, can be improved by data integration; for example, data from different providers can be not only visualised but merged and utilised in order to elaborate more complex products. A typical example can be an oil drift model, operated locally with meteorological input from another service provider.

This can be done by means of open source tools

\section{Acknowledgements}

The presented work has been carried out in the framework of two shared-cost research and technological development projects funded by the IST Programme: 
DISMAR, under Framework Programme (FP) 5 (Contract No. IST-2001-37657), and InterRisk, under FP6 (Contract No. 025253).

ERS SAR images are ESA Copyright.

\section{References}

[1] Espedal, H.A. \& Johannessen, O.M., Detection of oil spills near offshore installations using synthetic aperture radar (SAR). Int. J. Remote Sensing, 21, 11, 2141-2144, 2000

[2] Alpers, W. \& Espedal, H. E., Oils and Surfactants, Synthetic Aperture Radar Marine User's Manual. Eds: Jackson and Apel, NOAA, 2004

[3] Beal, R., Kudryavtsev, V., Thompson, D., Grodsky, S., Tilley, D., Dulov, V. \& Graber, H., The influence of the marine atmospheric boundary layer on ERS-1 synthetic aperture radar imagery of the Gulf Stream. J. Geophys. Res, 102, C3, 5799-5814, 1997

[4] Espedal, H.A., Oil spill and its look-alikes in ERS SAR Imagery. Earth Obs Rem. Sens., 16, 813-825, 2001

[5] http://www.eoportal.org/

[6] http://services.eoportal.org/

[7] Trivero, P., Fiscella, B., Gomez, F. \& Pavese, P., SAR detection and characterization of sea surface slicks, International Journal of Remote Sensing, 19, 543-548, 1998

[8] Trivero, P., Fiscella, B., \& Pavese, P., Sea surface slicks measured by SAR, Il Nuovo Cimento C, 1, 99-111, 2001

[9] Fiscella, B., Giancaspro, A., Nirchio, F., Pavese, P. \& Trivero, P., Oil spill detection using marine SAR images, International Journal of Remote Sensing, 21, 3561-3566, 2000

[10] Nirchio, F., Sorgente, M., Giancaspro, A., Biamino, W., Parisato, E., Ravera, R. \& Trivero, P., Automatic detection of oil spills from SAR images, International Journal of Remote Sensing, 26 (6), 1157-1174, 2005

[11] Hersbach, H., Stoffelen, A. \& de Haan, S., An improved scatterometer ocean geophysical model function: CMOD5, Journal of Geophysical Research, 5767-5780, 112, C03006, 10.1029/2006jc003743, 2007.

[12] Zecchetto, S., De Biasio, F. \& Trivero, P., Computation of wind direction from SAR images without external a priori information, Proc. of the Geoscience and Remote Sensing Symposium, 2007. IGARSS 2007 Barcelona. IEEE International, 3277-3280

[13] Fingas, M.F. and C.E. Brown, "Review of Oil Spill Remote Sensing", in Proceedings of Ecoinforma'96, Global Networks for Environmental Information, Environmental Research Institute of Michigan, Ann Arbor, Michigan, Vol. 10, pp. 465-470, 1996.

[14] Galeazzi, C. \& Portelli, C., The SkyMed/COSMO Platform Preliminary Definition, Proc. of the 4th International Symposium on Small Satellites Systems and Services, Sept. 14-18, 1998, Antibes Juan les Pins, France

[15] Amorosi, L, Notarantonio, A., Calio, E., Ritorto, A. \& Galleazzi, C., COSMO-Skymed constellation control: hardware and software 
implications, Proc. of IAC 2004, Vancouver, Canada, Oct. 4-8, 2004, IAC04-A.2.04

[16] Candela, L. \& Caltagirone, F., COSMO-SkyMed: Mission Definition, Main Applications and Products, Proc. of POLinSAR (Polarimetry and Polarimetric Interferometry) Workshop, ESA/ESRIN, Frascati, Italy, Jan. 14-16, 2003.

[17] Trivero, P., Biamino, W. \& Nirchio, F., High resolution COSMO - SkyMed SAR images for oil spills automatic detection, Proc. of the Geoscience and Remote Sensing Symposium, 2007. IGARSS 2007 Barcelona. IEEE International, $2-5$

[18] Ó Tuama, E. \& Hamre, T., Design and Implementation of a Distributed GIS Portal for Oil Spill and Harmful Algal Bloom Monitoring in the Marine Environment, Marine Geodesy, 30 (1-2), 145-168, 2007 